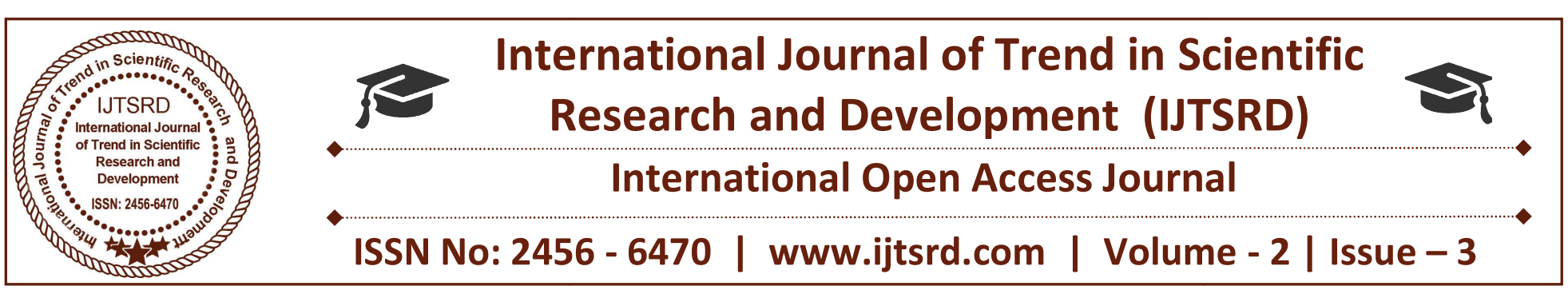

\title{
Techniques for Heat Transfer Augmentation in A Heat Exchanger:
}

\section{A Review}

\author{
Irsad Alam ${ }^{1}$, Prof. Rohit Soni ${ }^{2}$ \\ ${ }^{1}$ Research Scholar, ${ }^{2}$ Professor, \\ Trinity Institute of Technology and Research, \\ Rajiv Gandhi Proudyogiki Vishwavidyalay, Bhopal, Madhya Pradesh, India
}

\begin{abstract}
Many industrial application including power plants, chemical, refrigerator and air conditioning systems use heat exchangers to remove excess generated heat out of the system. Heat exchangers have several industrial and engineering applications. The design procedure of heat exchangers is quite complicated, as it needs exact analysis of heat transfer rate and pressure drop estimations apart from issues such as long-term performance and the economic aspect of the equipment. Heat transfer augmentation techniques refer to different methods used to increase rate of heat transfer without affecting much overall performance of the system. These techniques broadly are of three types viz. passive, active and compound techniques. The present paper includes various heat transfer augmentation techniques. A literature review of heat transfer augmentation has been included. This paper presents a review of published works on the heat transfer enhancement by active and passive methods.
\end{abstract}

Keywords: Heat Exchanger, Heat Transfer Augmentation, Active Techniques, Passive Techniques, Compound Techniques.

\section{INTRODUCTION}

Heat exchangers have several industrial and engineering applications. The design procedure of heat exchangers is quite complicated, as it needs exact analysis of heat transfer rate and pressure drop estimations apart from issues such as long-term performance and the economic aspect of the equipment. The major challenge in designing a heat

exchanger is to make the equipment compact and achieve a high heat transfer rate using minimum pumping power. Techniques for heat transfer augmentation are relevant to several engineering applications. In recent years, the high cost of energy and material has resulted in an increased effort aimed at producing more efficient heat exchange equipment. Furthermore, sometimes there is a need for miniaturization of a heat exchanger in specific applications, such as space application, through an augmentation of heat transfer. A heat exchanger is a device used to transfer heat between one or more fluids. The fluids may be separated by a solid wall to prevent mixing or they may be in direct contact. They are widely used in space heating, refrigeration, air conditioning, power stations, chemical plants, petrochemical plants, petroleum refineries, natural-gas processing, and sewage treatment. The classic example of a heat exchanger is found in an internal combustion engine in which a circulating fluid known as engine coolant flows through radiator coils and air flows past the coils, which cools the coolant and heats the incoming air, due to increased demands by industry for heat exchange equipment that is less expensive to build and operate than standard heat exchange devices. Recently, large numbers of attempts have been made to develop enhancement techniques to reduce the size and costs of heat exchangers in order to improve the overall performance of heat exchangers. An extensive literature survey of research on all types of enhancement technique is given in Webb and Bergles. 
1.1Generally, heat transfer augmentation methods are classified in three broad categories

(a) Active method: These techniques are more complex from the use and design point of view as the method requires some external power input to cause the desired flow modification and improvement in the rate of heat transfer. It finds limited application because of the need of external power in many practical applications.

(b) Passive method: These techniques generally use surface or geometrical modifications to the flow channel by incorporating inserts or additional devices. They promote higher heat transfer coefficients by disturbing or altering the existing flow behavior (except for extended surfaces) which also leads to increase in the pressure drop. These techniques do not require any direct input of external power; rather they use it from the system itself which ultimately leads to an increase in fluid pressure drop.

(c) Compound method: A compound augmentation technique is the one where more than one of the above mentioned techniques is used in combination with the purpose of further improving the thermo-hydraulic performance of a heat exchanger. When any two or more of these techniques are employed simultaneously to obtain enhancement in heat transfer that is greater than that produced by either of them when used individually, is termed as compound enhancement. This technique involves complex design and hence has limited applications

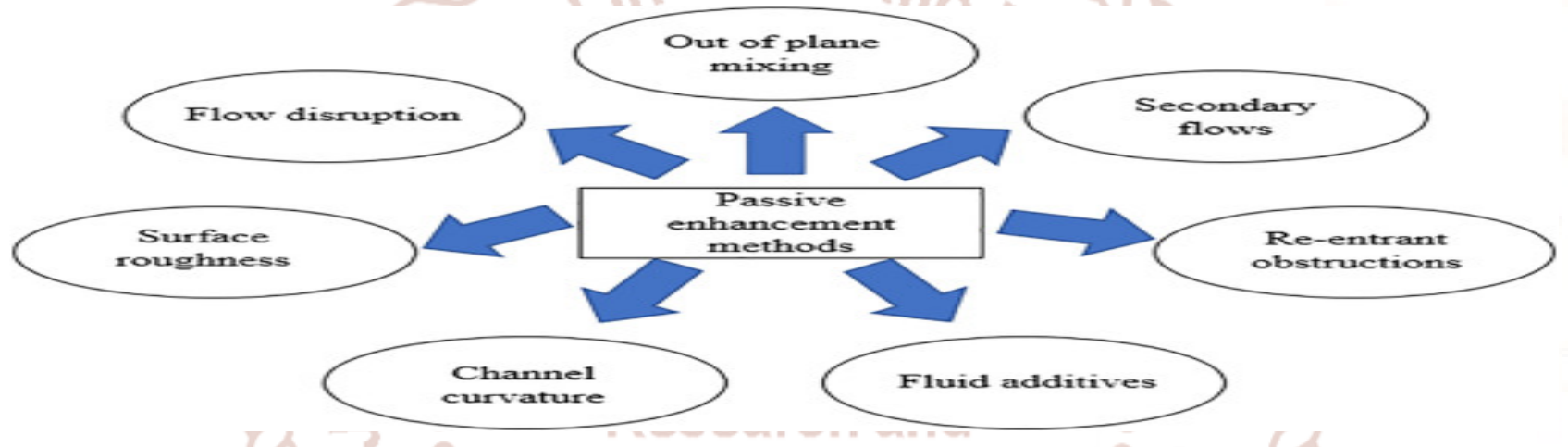

Figure 1. Passive Techniques for Heat Transfer Augmentation

\subsection{Heat transfer enhancement techniques in heat exchanger}

Enhancement of heat transfer using various techniques has received strong attention over the years in order to reduce the size and cost of heat exchanger. Many techniques have been developed for enhancing heat transfer rate in heat exchanger as the effective ones: (1) Nanofluids (2) Inserting fluid turbulators and (3) Roughening heat exchanger surfaces. Although for better heat transfer, combination of all the three or any two techniques can be used.

Heat exchanger using nano fluid is a device in which the heat transfer takes place by using nano fluid. In this the working fluid is nano fluid. Nano fluid is made by the suspending nano particles in the fluid like water, ethylene glycol and oil, hydrocarbons, fluorocarbons etc.

\subsubsection{Nano fluids}

Nanofluid, a suspension of nanoparticles in a continuous and saturated liquid, has been found capable to get considerably higher thermal conductivities than their respective base fluids resulting in better convective heat transfer coefficients. Fluids have higher specific heat compare to metals, and metals have higher thermal conductivity compare to solids. So when we added a small amount of nanoparticle to basefluid it will increase the thermal conductivity of basefluid.

The thermal conductivity of nanofluids has drawn increasing attention since first postulated that heat transfer could be improved through the addition of metallic nanoparticles to the heat transfer fluid. He addressed the limitation in thermal conductivity of typical heat transfer fluids and suggested the addition of more conductive solid particles would enhance the fluid thermal conductivity beyond that suggested by conventional models. The advantages of using 
nanoparticles are that they are more easily suspended in the fluid, they may be used in micro channels, and the small size causes less wear to machinery. However, aggregation of particles must be minimized in order to benefit from these effects of small particle size.

\subsubsection{Fluid turbulators}

Heat transfer can also be enhanced by using rotating inserts in a round tube. These rotating insert acts as a swirl generator. The use of the swirl generator is expected to create the tangential velocity or swirling flow to prolong residence time of the flow and to enhance the tangential and radial fluctuation, therefore leading to increase in heat transfer inside the test tube .These techniques are more complex from the use and design point of view as the method requires some external power input to cause the desired flow modification and improvement in the rate of heat transfer. It finds limited application because of the need of external power in many practical applications. In comparison to the passive techniques, these techniques have not shown much potential as it is difficult to provide external power input in many cases. In these cases, external power is used to facilitate the desired flow modification and the concomitant improvement in the rate of heat transfer. Augmentation of heat transfer by this method can be achieved by:

\subsubsection{Mechanical Aids:}

Such instruments stir the fluid by mechanical means or by rotating the surface. These include rotating tube heat exchangers and scrapped surface heat and mass exchangers.

\subsubsection{Surface vibration:}

They have been applied in single phase flows to obtain higher heat transfer coefficients.

\subsubsection{Fluid vibration:}

These are primarily used in single phase flows and are considered to be perhaps the most practical type of vibration enhancement technique.

\subsubsection{Electrostatic fields:}

It can be in the form of electric or magnetic fields or a combination of the two from dc or ac sources, which can be applied in heat exchange systems involving dielectric fluids. Depending on the application, it can also produce greater bulk mixing and induce forced convection or electromagnetic pumping to enhance heat transfer

\subsubsection{Injection:}

Such a technique is used in single phase flow and pertains to the method of injecting the same or a different fluid into the main bulk fluid either through a porous heat transfer interface or upstream of the heat transfer section.

\subsubsection{Suction:}

It involves either vapor removal through a porous heated surface in nucleate or film boiling, or fluid withdrawal through a porous heated surface in singlephase flow.

\subsubsection{Jet impingement:}

It involves the direction of heating or cooling fluid perpendicularly or obliquely to the heat transfer surface

\subsubsection{Geometrical modification (by roughening heat exchanger surfaces)}

These techniques generally use surface or geometrical modifications to the flow channel by incorporating inserts or additional devices. They promote higher heat transfer coefficients by disturbing or altering the existing flow behavior (except for extended surfaces) which also leads to increase in the pressure drop. In case of extended surfaces, effective heat transfer area on the side of the extended surface is increased. Passive techniques hold the advantage over the active techniques as they do not require any direct input of external power. These techniques do not require any direct input of external power; rather they use it from the system itself which ultimately leads to an increase in fluid pressure drop. They generally use surface or geometrical modifications to the flow channel by incorporating inserts or additional devices. They promote higher heat transfer coefficients by disturbing or altering the existing flow behavior except for extended surfaces. Heat transfer augmentation by these techniques can be achieved by using;

\subsubsection{Treated Surfaces:}

Such surfaces have a fine scale alteration to their finish or coating which may be continuous or discontinuous. They are primarily used for Boiling and condensing duties. 


\subsubsection{Rough surfaces:}

These are the surface modifications that promote turbulence in the flow field in the wall region, primarily in single phase flows, without increase in heat transfer surface area.

\subsubsection{Extended surfaces (Fins):}

They provide effective heat transfer enlargement. The newer developments have led to modified finned surfaces that also tend to improve the heat transfer coefficients by disturbing the flow field in addition to increasing the surface area.

\subsubsection{Displaced enhancement devices:}

These are the inserts that are used primarily in confined forced convection, and they improve energy transport indirectly at the heat exchange surface by displacing the fluid from the heated or cooled surface of the duct with bulk fluid from the core flow.

\subsubsection{Swirl flow devices:}

They produce and superimpose swirl flow or secondary recirculation on the axial flow in a channel. These include helical strip or screw type tube inserts, twisted tapes. They can be used for single phase and two-phase flows.

\subsubsection{Coiled tubes:}

These lead to relatively more compact heat exchangers. It produces secondary flows and vortices which promote higher heat transfer coefficients in single phase flows as well as in most regions of boiling

\subsubsection{Surface tension devices:}

These consist of wicking or grooved surfaces, which direct and improve the flow of liquid to boiling surfaces and from condensing surfaces.

\subsubsection{Additives for gases:}

These include liquid droplets or solid particles, which are introduced in single- phase gas flows either as dilute phase (gas-solid suspensions) or as dense phase (fluidized beds).

\subsection{Conclusions}

This chapter covers brief summery about the heat exchangers and potential for increasing the heat transfer rate. From the above techniques we find that introduction of nanofluids and extended surfaces (fins) may be the possible methods which can be used at the same time for heat transfer augmentation.

\section{LITERATURE REVIEW}

As the need for more efficient heat transfer systems increases, researchers have resorted to various heat transfer enhancement techniques since the mid-1950s. The significant increase in the number of research articles dedicated to this subject thus far shows a noticeable growth and the importance of heat transfer enhancement technology.

A considerable amount of experimental as well as analytical and computational research has been carried out on the enhancement of heat transfer. In this chapter, a brief survey of the relevant literature is presented to indicate the extent of work already reported in open literature pertaining to the enhancement of heat transfer by introducing protrusions mounted on the heat transfer surfaces.

Grosse-Gorgemann et al. [1993] showed that the enhancement mechanism by transverse vortex generators need unsteady flow and develop reversed flow regimes which further increase the resistance to flow. No enhancement in heat transfer was reported for steady flow in a periodically ribbed channel.

Ghaddar et al. [1986] and Amon and Mikic [1990] investigated numerically the grooved channel flow where the grooves were so short that the separated flow was attached at the face of the next protrusion instead of the base of the groove.

Turk et al. [1986] investigated heat transfer enhancement for laminar flow over a row of rectangular winglet pairs by varying the aspect ratio. The angle of attack was fixed at $20^{\circ}$. It was found that the ratio of span averaged heat transfer coefficient on a flat plate with vortex generator to the corresponding value without vortex generator increased up to 3 at a distance more than 30 chord lengths downstream of the winglets. The study was carried out both for zero and favorable pressure gradients and heat transfer enhancement was found to be more with favorable pressure gradient.

Torii et al. [1991] investigated local heat transfer downstream of a single delta winglet vortex generator on a flat plate. Flow visualization experiments were conducted to study the flow field and hot wire 
anemometer was used to measure the velocities. Naphthalene sublimation and surface thermocouples with an imposed heat flux were used to measure the heat transfer. The free stream velocity was fixed to 4 $\mathrm{m} / \mathrm{s}$. Local heat transfer enhancement of over $200 \%$ was reported in the downwash region of the flow. The velocity data provided the information about the vortex location.

Gentry and Jacobi [1997] studied the effect of stream wise vortices induced by delta wing vortex generator. Flow visualization techniques were used to study the flow and naphthalene sublimation was used to get the heat transfer effects. It was concluded that the maximum heat transfer enhancement is observed when a vortex is located near the edge of the thermal boundary layer. Further it was reported that the vortices should be generated in a common inflow arrangement so that the induced velocities keep the vortices near the boundary layer.

Prabhanjan et al. [2002] Authors found that many industries use helical coil tube heat exchanger due to its higher heat transfer coefficients than straight tube heat exchanger due to increased buoyancy effects. A comparative study was undertaken to determine heat transfer coefficient. Flow rate did not affect the heat transfer coefficient, most likely from the fact that the flow was turbulent and increasing the flow rate does not greatly change the wall effects. Author also found that the temperature rise of the fluid was found to be effected by coil geometry and by the flow rate.

Salimpour [2009] investigated experimentally the heat transfer coefficients of shell and helically coiled tube heat exchangers with different coil pitches were selected as test section for both parallel-flow and counter flow configurations. It was found that Nusselt numbers increase with Reynolds number. It was revealed that the correlation for constant temperature boundary conditions quite in agreement with the present data in low Dean number region. From the results of the present study, Author was found out that the shell-side heat transfer coefficients of the coils with larger pitches are higher than those for smaller pitches. Finally, based on the results of this study, two correlations were developed to predict the inner and outer heat transfer coefficients of the coiled tube heat exchangers.

Eed Abdel Hafez Abdel-hadi et al.[2006]: Investigated the heat transfer analysis of vapour compression system using CuO-R134a Nano fluid, test section made of copper horizontal tube and heat is applied 10-40 KW/m ${ }^{2}$. Heat flux, concentration, and size particle is important factor to enhance the heat transfer rate of Nano fluid. Heat transfer rate is increases with increasing heat flux, up to $55 \%$ of concentration of Nano fluid and up to $25 \mathrm{~nm}$ sized particles.

Somchai wong wises et al.[2007]: Investigated heat transfer enhancement and flow characteristic of $\mathrm{Al}_{2} \mathrm{O}_{3}$-Water Nano fluid using micro channel heat sink. The dimension of test section is $5 \times 5 \mathrm{~mm}$ and $50 \mathrm{~W}$ heat is applied. Heat transfer is enhanced at high Reynolds number and high concentration of Nanofluid, because at high Reynolds number wall temperature is decreases and pressure drop is increased.

Yannar et al.[2008]: Investigated the flow and heat transfer characteristic of spiral pipe heat exchanger using different type of Nano fluid with different concentration such as $\mathrm{Al}_{2} \mathrm{O}_{3}$-water, $\mathrm{TiO}_{2}$-water, $\mathrm{CuO}$ water Nano fluid with $1 \%, 1 \%$ and $3 \%$ concentration respectively. Test section made of copper tube had the ratio of pitch per diameter is 7 , mean hydraulic diameter is $30 \mathrm{~mm}, 10 \mathrm{~mm}$ diameter and $1600 \mathrm{~mm}$ length. Heat transfer enhanced $28 \%$ at $0.8 \%$ concentration of Nano fluid, due to high concentration shear stress of Nano fluid is increased. Heat transfer enhancement is high in spiral pipe compared with circular pipe, because the pressure drop is high in spiral pipe. Heat transfer co efficient is decreases when axial distance of Nano fluid is increasing, because formation of boundary layer.

Manag et al.[2011]: Investigated the friction factor and heat transfer rate of $\mathrm{CuO}$-Water and $\mathrm{Al}_{2} \mathrm{O}_{3}$-water Nano fluid using micro channel heat sink. Dimension of test section is $100 \times 100 \times 20000$ micrometter, assumed steady state laminar flow occurred, neglected the radioactive heat transfer and adiabatic constant heat flux applied at bottom of heat sink is 5000 $\mathrm{W} / \mathrm{cm}^{2}$. As the result of increasing nusselt number with increasing the Reynolds number and concentration but decreased the friction factor of Nano fluid. Compared the $\mathrm{CuO}$-water and $\mathrm{Al}_{2} \mathrm{O}_{3}-$ water Nano fluids the $\mathrm{CuO}$-water Nano fluid showed better enhancement and low friction factor. 
K.Somasekhar et al.[2016]: In this study a multi pass shell and tube heat exchanger with 3 tubes modeling is done using CATIA and meshing has done using ICEM CFD software, simulations has done by using CFD-FLUENT software. Using Fluent, computational fluid dynamics software the pressure drop, heat transfer characteristics of $\mathrm{Al} 2 \mathrm{O} 3$-water nanofluid, and Distilled water are analyzed under turbulent flow condition. Nanofluid such as $\mathrm{Al}_{2} \mathrm{O} 3-\mathrm{H} 2 \mathrm{O}$ is used as cooling medium instead of Distilled water. Finally the CFD simulated results are compared with experimental results. The effects of Peclet number, volume concentration of suspended nanoparticles, and particle type on the heat transfer characteristics were investigated. Based on the results, adding of nanoparticles to the base fluid (Distilled water) causes the significant enhancement of heat transfer characteristics.

\section{CONCLUSIONS}

From the above literature survey it is conclude that :

a) Use of Nanofluids increases the heat transfer rate because of higher thermal conductivity.

b) Use of fins also help in increasing the heat transfer rate.

c) Heat transfer rate increases with increasing diameter of tube with curvature ratio

d) CNT Coating on substrate enhance in heat flux, surface area, increase in the surface roughness and without increase in the pressure drop.

e) Introduction of injection of air bubbles also help in increasing the heat transfer rate.

\section{REFERENCES}

1) Grosse-Gorgemann, A., Hahne, W., and Fiebig, M., 1993, "Influence of Rib Height on Oscillations, Heat Transfer and Pressure Drop in Laminar Channel Flow," Proceedings of Eurotherm 31, Vortices and Heat Transfer, Bochum, Germany, pp 36-41.

2) Mohd. Rehan Khan and Dr. Ajeet Kumar Rai. An Experimental Study of Exergy in A Corrugated Plate Heat Exchanger, International Journal of Mechanical Engineering and Technology, 6(11), 2015, pp. 16-22.

3) Ghaddar, N. K., Korzak, K. Z., Mikic, B. B. and Patera, A. T., 1986, "Numerical Investigation of
Incompressible Flow in Grooved Channels, Part 1, Stability and Self Sustained Oscillations," Journal of Fluid Mechanics, Vol. 163, pp. 99-127.

4) Turk, A.J, and Junklan, G.H., 1986, "Heat Transfer Enhancement Downstream of Vortex generators on a Flat Plate," Proceedings of the Eighth International Heat Transfer Conference, San Francisco, Vol. 6, pp. 2903-2908.

5) Torii, K., Yanagihara, J. I., and Nagai, Y., 1991, "Heat Transfer Enhancement by Vortex Generators," Proceedings of the ASME/JSME Thermal Engineering Joint Conference, J. R. Lloyd, and Y. Kurosaki, eds., ASME Book No. I0309C, ASME, New York, pp. 77-83.

6) $\mathrm{T} K \mathrm{~S}$ Sai Krishna, S G Rajasekhar and C Pravarakhya. Design and Analysis of Plate Heat Exchanger with $\mathrm{Co} 2$ and R134a As Working Fluids, International Journal of Mechanical Engineering and Technology, 4(4), 2014, pp. 311318.

7) Gentry, M. C., and Jacobi, A. M., 1997, "Heat Transfer Enhancement by DeltaWing Vortex Generators on a Flat Plate: Vortex Interactions with the Boundary Layer,' Experimental Thermal and Fluid Science, Vol.14,pp.231-242.

8) D. G. Prabhanjan, G. S. V. Ragbavan and T. J. Kennic, Comparison of heat transfer rates between a straight tube heatexchanger and a helically coiled heat exchanger, lnt. Comm. Heat \& mass Trans. Vol. 29. No. 2. 2007, 185-191.

9) M.R. Salimpour Heat transfer coefficients of shell and coiled tube heat exchangers,Experimental Thermal and Fluid Science 33, 2009, 203-207. 